\title{
Determination of Serum Bilirubin by Skin Reflectance: Effect of Pigmentation
}

\author{
R. E. HANNEMANN, D. P. DEWITT, E. J. HANLEY, R. L. SCHREINER, PAULINE BONDERMAN \\ Biomedical Engineering Center, Purdue University, West Lafayette, Indiana, and Department of Pediatrics, Indiana \\ University School of Medicine, and the James Whitcomb Riley Hospital for Children, Indianapolis, Indiana, USA
}

\begin{abstract}
Summary
A noninvasive optical technique by which serum bilirubin can be estimated from skin spectral reflectance measurements has been further investigated. The original work on 30 healthy, full-term white infants and an independent study on 14 white and 30 black infants demonstrate that the method has potential not only for clinical use, but also for the study of the transport of bilirubin to, from and within the skin. The objectives of the present study are to evaluate the method on a larger sample population with special attention to natural skin pigmentation effects and the development of a physical model of the tissue to explain the relationship between serum bilirubin concentration and skin reflectance.

Reflectance spectra $(380-800 \mathrm{~nm})$ and concurrent serum bilirubin measurements were taken on a sample population of 58 white and 45 full-term black infants (1-3 days of age). Multiple linear regression analysis, comprised of six wavelengths gave a correlation coefficient, $\boldsymbol{r}=\mathbf{0 . 8 3 1}$ for the white infant group. For the black infant group, a five wavelength analysis provided $r=$ 0.877 with the standard error of estimate being $\pm 1.46 \mathrm{mg} / 100 \mathrm{ml}$ for both groups.

The model for establishing a physical basis for the relationship shows that a transformed, normalized Kubelka-Monk function $\xi$ $(460,510,420)$ is linearly related to serum bilirubin concentration. This function is determined from the spectral reflectance values at three wavelengths, 420,460 , and $510 \mathrm{~nm}$. The wavelength combination is such that effects due to hemoglobin and melanin pigments are minimized. Regression analysis showed that $r=$ 0.778 and $r=0.865$ for the white and black infant groups, respectively, with standard error of estimates being $\pm 1.4 \mathrm{mg} / 100$ ml for both groups.

Routine determinations of total serum bilirubin by laboratory methods have standard errors of estimate ranging from \pm 1 to 1.5 $\mathrm{mg} / 100 \mathrm{ml}$. Thus, the method herein described shows that the relationship between skin reflectance and serum bilirubin in fullterm infants is close to the acceptable limits for clinical use. Furthermore, this work shows that skin pigmentation does not obscure this relationship.
\end{abstract}

\section{Speculation}

Because skin pigmentation does not obscure the relationship between serum bilirubin and spectral reflectance, the latter is clinically useful for jaundice screening for all neonates. It may also be helpful in monitoring phototherapy of jaundiced infants although the spectral considerations may be extremely complex in these cases. Once such relationships are determined, however, skin reflectance may also offer a measure of the risks of kernicterus.

The role of bilirubin in the production of neonatal central nervous system damage is well established. The precise serum levels at which such damage commences is unknown but broad guidelines have been established for the initiation of treatment by exchange transfusion or phototherapy (19). Early detection and continual monitoring of elevated bilirubin levels is essential if kernicterus is to be prevented. Although serum tests are relatively accurate and easily done, they are often delayed because of failure of visual detection of jaundice and the reluctance to perform invasive procedures particularly on the very small premature.

To obviate these difficulties, a noninvasive optical technique was developed by which the reflectance at specific wavelengths could be used to determine the total serum bilirubin concentration. The original investigative work, previously reported by the authors (8), was performed on a sample of 30 caucasian, healthy, full-term infants. The results demonstrated that there was a strong relationship between skin reflectance and serum bilirubin concentration and that the method had potential not only as a research device for study of the blood-serum bilirubin transport system, but also as a tool for use in clinical screening for jaundice. This original work was followed by a recent, independent study (16) on 14 white and 30 black infants producing similar results although details of the method have not been published. However, because of the small sample population and the lack of data on natural skin pigmentation effects, in both of these studies, the generality of the method could not be fully tested. Hence, the present study was performed with the objectives to evaluate the method on a large sample of both black and white infants and to develop a physical model explaining the relationship between skin optical properties and the serum bilirubin concentration.

\section{MATERIALS AND METHODS}

The patient selection process and measurement procedures followed protocols that were approved for use by the Human Subjects Committee of Purdue University and the Clinical Research Committee of Indiana University. Reflectance spectra and concurrent serum bilirubin measurements were taken on a sample population consisting of 58 white and 45 black, full-term, infants ranging from 1-3 days of age.

The spectral reflectance measurements on the infant's skin were made using the reflectometer developed at Purdue University and shown in Figure 1. The major components include: tungsten light source (General Electric, Quartzline Lamp, Model 1945) and power supply (Sorenson, Model DCR 60-13A); bifurcated fiber optic bundle (Ealing, Model 22-0533) mating the source, probe, and spectroradiometer; spectroradiometer system consisting of a Wadsworth type spectrometer with flint glass prism using a silicon detector, scan speed range $1-32 \mathrm{sec} / \mathrm{scan}$, and a wavelength range $380-800 \mathrm{~nm}$ with a spectral resolution typically better than $2 \%$; and data acquisition system consisting of a four-channel FM tape recorder (Hewlett Packard 3968-A). Two channels of the tape recorder record detector level and wavelength positions signals. The third channel is used for test/monitor signals (such as indicating whether the detector signal is from a subject, reference standard, etc.); the fourth channel is used to record audio information such as patient identification, procedures, and other data that might prove useful in subsequent analysis of the clinical 


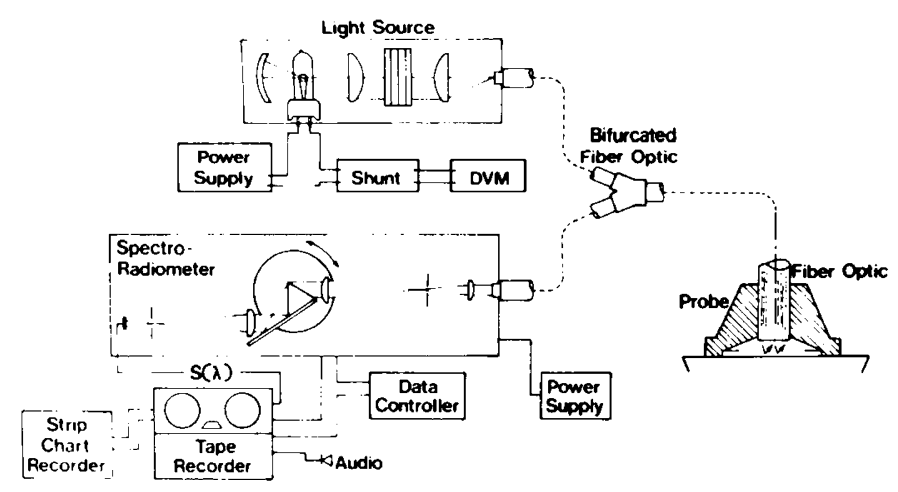

Fig. 1. Schematic of the reflectometer system for skin reflectance measurements.

observations. A two-channel strip chart recorder (MFE Model $\mathrm{M} 22 \mathrm{C}$ ) provides the means to visually monitor proper operation of the system and the sequence of measurement.

An important aspect of the measurement procedure is the data processing of the clinical observations. To convert the analog measurements recorded on the tape recorder to digital format suitable for computer analysis, a processing system, called EXOSYS (18) was used. In the first step, the analog data (detector signal) is converted to 10 bit binary data. Wavelength positions of the spectroradiometer are determined from a spectral calibration gauging curve based upon a fifth-order, polynomial fit to the calibrating spectral lines. Detector signals from the subject's skin are compared to signals from a reference standard, barium sulphate $\left(\mathrm{Ba}_{2} \mathrm{SO}_{4}\right)$. The resulting spectral reflectance (relative to the reference and converted to an absolute basis) of the subject is then stored along with identification and clinical observations (such as serum bilirubin and other tests) on magnetic tapes.

The research reflectometer system was set up, tested, and calibrated in a nursery environment in $20 \mathrm{~min}$. The body-site location found to be most reproducible was the posterior mid thorax and measurements were taken on both the left and right sides. The reflectometer probe, illustrated in Figure 1 , has a $2 \mathrm{~cm}$ diameter and irradiates a $\mathbf{l ~ c m}^{2}$ area with a total radiant power less than 20 $\mathrm{mW}$.

Immediately after the reflectance spectra measurement total serum bilirubin was determined by the Jendrassik method (6). Other parameters which were entered into the data log for each infant included: time of measurement, age, estimated gestational age, sex, height, and weight. Single measurements of total protein, albumin, globulin, and hematocrit were also taken. Observations were done between $10 \mathrm{AM}$ and $1 \mathrm{PM}$, and between $2 \mathrm{PM}$ and 5 PM in order to avoid disturbances to the routine nursery schedule.

\section{RESULTS}

A composite summary of the 220 reflectance spectra, $400-760$ $\mathrm{nm}$ wavelength range, for the 58 white and $\mathbf{4 5}$ black infant groups is shown in Figure 2. The spectra averaged for three ranges of serum bilirubin concentration showed the expected spectral behavior due to increased bilirubin concentration levels and to pigmentation. As earlier investigators $(1,13,17)$ demonstrated, it is not possible to infer concentration levels directly from the spectra. Rather, it is necessary to perform a multispectral analysis wherein an interrelationship between selected spectral reflectance values and concentration level can be found. Using this data set, two methods of analysis-statistical spectral correlation and physical modeling-were used.

\section{STATISTICAL SPECTRAL CORRELATION}

The first goal of this study was to statistically analyze relationships for predicting bilirubin level from various combinations of the spectral reflectance data set. The correlations considered were based upon: reflectance in a single wavelength band, ratio of the

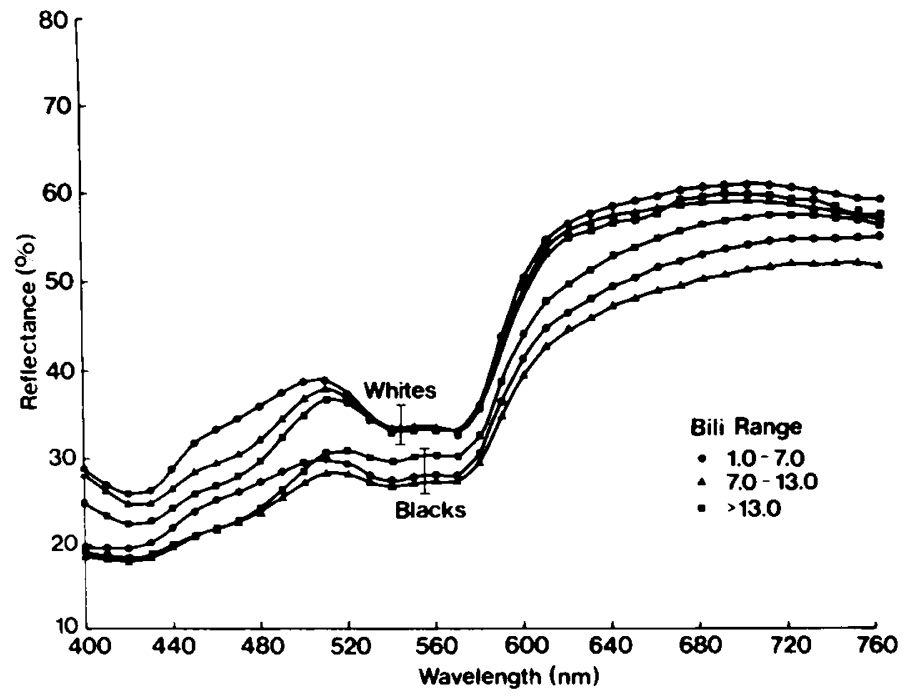

Fig. 2. Representative reflectance spectra for ranges of bilirubin concentration ( $\mathrm{mg} / 100 \mathrm{ml}$ serum) based upon sample populations of 58 white and 45 black full-term infants.

reflectance in two wavelength bands, and multiple linear regressions.

The result of a linear regression analysis showed that reflectance in a single wavelength band was inadequate for prediction of bilirubin level. At most, 21 and $5 \%$ of the variation in the bilirubin concentration could be explained for the white and black infants, respectively. As expected, the spectral wavelength of most significance was $460 \mathrm{~nm}$, which corresponds to the absorption band of bilirubin. Regression analysis based upon the ratio of two reflectance values significantly increased the correlation coefficient, $r$. For the white group, a correlation based upon the ratio of reflectance at $450 \mathrm{~nm}$ to the reflectance at $520 \mathrm{~nm}$, yielded $r=0.81$. For the black infant group, the reflectance ratio, $480 \mathrm{~nm} / 500 \mathrm{~nm}$, proved to be the best combination with $r=0.83$. It was evident that the relationship for the black group was slightly better than for the white group. This indicated that pigmentation effects prominent in the blacks did not obscure jaundice. While the reflectance serum bilirubin concentration relationships were of the same form of both groups, the constants were sufficiently different that it was appropriate to consider the groups separately.

Based upon the experiences of our earlier study (8), it is expected that relationships based upon multiple-linear regressions would have increased $r$ values because more spectral information is used to explain the serum bilirubin concentration variation. Using the 41 spectral reflectance data set, a best subset of spectral values for prediction of bilirubin was selected for each infant race group using a combination of regression techniques. For the white group, the optimal subset was comprised of six wavelengths $(410,420$, $440,490,520$, and $550 \mathrm{~nm}$ ) giving an $r$ value of 0.831 and a standard error of estimate $\pm 1.45 \mathrm{mg} / 100 \mathrm{ml}$. For the black group, the optimal subset was five wavelengths $(400,490,520$, and 590 $\mathrm{nm})$ giving $r=0.877$ with the standard error of estimate being $\pm 1.47 \mathrm{mg} / 100 \mathrm{ml}$. As anticipated, the $r$ values for the multiple wavelength analysis were greater than those for the ratio-reflectance analysis. However, the results from the statistical analysis did not provide insight into the causal relationship between skin reflectance and bilirubin concentration. That is, the form of the correlation and the subset of wavelengths used are based upon statistical rather than physical significance.

\section{DEVELOPMENT OF THE PHYSICAL MODEL}

The second part of the study was directed toward establishing a physical basis for a relationship between skin reflectance and serum bilirubin. The model developed (7) for this purpose assumes the skin to be a one-dimensional, homogeneous medium having an absorption coefficient, $w$, and a scattering coefficient, $k$. Based 
upon diffusion theory analysis, these optical properties can be related to the skin surface reflectance, $\rho$, according to the relation:

$$
\xi=\frac{\mathrm{w}}{\mathrm{k}}=\frac{\rho}{2}+\frac{1}{2 \rho}-1
$$

where $\xi$ is referred to as the Kubelka-Munk (K-M) function (12).

It was assumed that the tissue is comprised of several uniformly distributed constituents and that the bulk optical properties were linear combinations of the properties of the constituents. Only the dominant constituents bilirubin (b), hemoglobin (h), and melanin (m) were considered. The model isolates the absorption coefficient of bilirubin, $w_{b}$, which from Beers' law is linearily related to the concentration of bilirubin, $\mathrm{C}_{\mathrm{b}}$, with the result:

$$
\bar{\xi}(460,510,420)=\frac{\xi_{460}-\xi_{510}}{\xi_{420}}=a+b \cdot C_{b}
$$

The numerical subscripts designate a spectral value at some wavelength (nm) and the coefficients, $a$ and $b$, are constants depending upon the volume fractions of hemoglobin and melanin in the skin. This model suggests that this optical parameter, $\bar{\xi}$, dependent upon the spectral reflectance of three specific wavelengths, is linearily dependent upon the concentration of bilirubin in the serum. This combination of wavelengths minimizes the influence of natural variations in relative oxygen saturation, vascularity, and melanin pigment content in the skin such that a general relation between serum bilirubin and the skin reflectance is possible.

To determine the reliability of the physical model predicting a linear relationship between the K-M function, $\bar{\xi}(420,460,520)$, and bilirubin concentration, $C_{b}$, a regression analysis was performed. For the combined black and white infant population, the correlation coefficient, $r$, was 0.706 with a standard error of estimate of $\pm 1.6 \mathrm{mg} / 100 \mathrm{ml}$. This relationship can be improved by separating the infant population into the two race groups, thus, reducing the effect of variation in melanin pigment within each group. For the white group (58 infants, 124 observations), $r=$ 0.778 and the standard error of estimate is $\pm 1.4 \mathrm{mg} / 100 \mathrm{ml}$. Figure 3 shows the comparison of the $K-M$ function, $\bar{\xi}$, with the serum bilirubin concentration laboratory measurements. The black group, (45 infants, 96 observations), Figure 4, was found to be more highly correlated with $r=0.865$ and a standard error of estimate of $\pm 1.4 \mathrm{mg} / 100 \mathrm{ml}$. This higher correlated behavior by the black group can be attributed to one or more of the following: 1) the degree of pigment variability is not as large within the black group as within the white group; 2) melanin, more predominant in the black group, prevents light penetration to the deeper dermal layers where a variety of undesirable absorption and scattering mechanisms may be encountered, and 3 ) the melanin pigment acts as a filter which obscures the detailed scattering and absorbing mechanism locally and thus has a homogenizing effect. By comparison of Figures 3 and 4, it can be seen that the slope of the

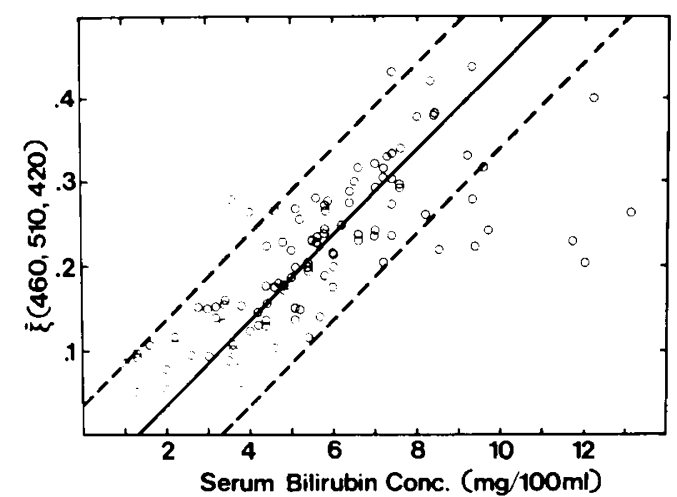

Fig. 3. Transformed, normalized Kubelka-Munk function, $\bar{\xi}$, based upon spectral reflectance values at 460,510 , and $420 \mathrm{~nm}$ as a function of measured serum bilirubin concentration $(\mathrm{mg} / 100 \mathrm{ml})$ for the white fullterm infant sample.

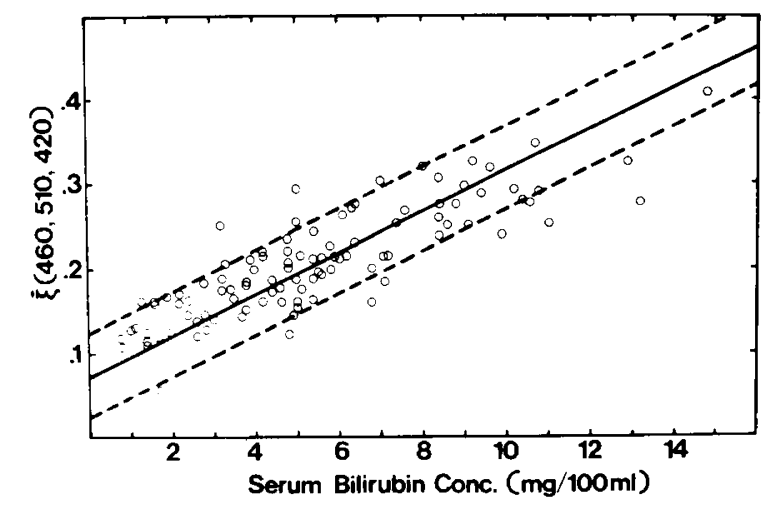

Fig. 4. Transformed, normalized Kubelka-Munk function, $\bar{\xi}$, based upon spectral reflectance values at 460,510 , and $420 \mathrm{~nm}$ as a function of measured serum bilirubin concentration $(\mathrm{mg} / 100 \mathrm{ml})$ for the black fullterm infant sample.

correlation (linear) curve for the black group is less than that for the white group. This effect is due to increased melanin absorption at $420 \mathrm{~nm}$ in the black group.

\section{DISCUSSION}

\section{OPTICAL PROPERTIES OF SKIN}

There exists a limited body of knowledge concerning the optical properties of jaundiced skin. To understand more thoroughly the phenomenon of how light interacts with the skin, it would be desirable to have the reflectivity (or absorptivity), scattering coefficient, and absorption coefficient as a function of wavelength, primarily in the range $380-800 \mathrm{~nm}$ for skin in the normal and jaundiced conditions. The most significant optical feature of jaundiced skin is the spectral absorptance at $465 \mathrm{~nm}$ which is a characteristic of the bilirubin compound. Sheard and Brown (17) obtained the first reliable spectral reflectivity of the human skin in vivo in the visible spectral region. From that time, several other investigators $(10,13)$ using commercial spectrophotometers with integrating sphere attachments of similar design, have established that human skin reflectance spectra is dependent upon skin texture, pigmentation and body location.

Ballowitz and Avery (1), also using a commercial spectrophotometer, made the first reflectance spectra measurements on normal and jaundiced infants. They made "one-time" observations showing the effect excess bilirubin has on the reflectance spectra. Particularly important is the decrease of the reflectance in the $400-500 \mathrm{~nm}$ region (near the absorption band of bilirubin) as the serum bilirubin increases. To the eye, this would give rise to a yellow skin appearance. Their limited data indicated that the variability in normal infant pigmentation would obscure any quantitative conclusions regarding reflectance changes with bilirubin level. The clinical experiences of the authors indicate that handling of the infant (necessary to position the skin surface at the sample port of the spectrophotometer integrating sphere) can cause large changes in the reflectance spectra. Therefore, in order to obtain reliable data, it is necessary to disturb the infant as little as possible.

Krauss et al. (11) investigated the skin reflectance of 99 healthy newborn white and black infants ranging in gestational age from 26-46 wk. They used a commercial portable reflectometer equipped with three standard filters: blue, green, and red. The information presented on these filters, however, is inadequate for relating their observations to our spectral reflectance measurements or those of Ballowitz and Avery (1). However, their study does present qualitative information on reflectance changes with maturation of the infant. Krauss et al. (11) speculate that reflectance spectrometry may be useful to determine effects of various therapeutic regimens on the skin. The work of the authors would support this speculation, however, it is believed to be important to perform spectral rather than broad-band reflectance measure- 
ments. Color analysis, such as others have performed, is insensitive to subtle changes in spectral reflectance in narrow wavelength bands because the spectrum is essentially averaged over wide spectral regions. Brunsting and Sheard (3) reached this conclusion in 1929 by noting that jaundice is qualitatively detectable from the reflectance spectra but is not noticeable when using a color analysis scheme. The limited available literature on reflectance spectrometry, particularly as related to infants, indicates that the spectral (rather than color or broad-band) analysis is the preferred method for studying disease-oriented phenomena through skin observations.

The optical properties of serum bilirubin in vitro have been extensively studied. Most recently, Lee and Gartner (15) have measured the spectrophotometric characteristics at low concentrations under various physical conditions. For use in our modeling studies, the absorption coefficient as measured by Hertz et al. (9) appears to be the most representative for relating skin reflectance to cutaneous bilirubin concentration. The absorption coefficients for oxyhemoglobin $\left(\mathrm{HbO}_{2}\right)$ and hemoglobin $(\mathrm{Hb})(20)$ have spectral character that exert a large influence on the spectral reflectance of skin. The other important constituent is the pigment melanin (2) which has some spectral character but no strong absorption peaks.

\section{SIGNIFICANCE OF THE PRESENT STUDY}

The data presented in this study show that there is a strong relationship in both white and black full-term infants between serum bilirubin concentration and skin reflectance. Furthermore, it shows that the pigmentation of the skin does not obscure the relationship. Preliminary results from another institution are supportive of these findings (16).

The most favorable relationships for both racial groups gives a standard error of estimate of $\pm 1.5-2.0 \mathrm{mg} / 100 \mathrm{ml}$. Routine determinations of total serum bilirubin by laboratory methods have standard errors of estimate ranging from \pm 1 to $1.5 \mathrm{mg} / 100 \mathrm{ml}(4$, 14). Commercially available spectrophotometers designed specifically for measuring total bilirubin level may have a limit of error even higher than $\pm 1.5 \mathrm{mgm}$ (5). Thus, the relationship between skin reflectance and serum bilirubin in full-term infants is relatively close to acceptable limits for clinical methods now in use. Although a clinical tool is possible, the use of such an instrument for furthering basic knowledge in bilirubin transport and deposition under normal phototherapy, and exchange transfusions is even more exciting and potentially valuable. These studies are planned. Of additional significance is the possibility that further studies could reveal a relationship between skin reflectance and the risk for kernicterus. Research in this most important area will have to await the development of the interrelationships of the diffusion characteristics of unbound bilirubin across central nervous system and epidermal cell membranes.

\section{REFERENCES AND NOTES}

1. Ballowitz L and Avery, M. E.: Spectral reflectance of the skin. Biol. Neonate, 15: 348 (1970).

2. Blois, M. D.: On the spectroscopic properties of some natural melanins. J. Invest. Dermatol., 47: 162 (1966).

3. Brunsting, L. A., and Sheard. C.: The color of the skin as analyzed by spectrophotometric methods. II. The role of pigmentation. J. Clin. Invest., 7: 575 (1929).

4. Doumas. B. T., Perry. B. W.. Sasse. E. A.. and Straumfjord, J. V.. Jr.: Standardization in bilirubin assays: evaluation of selected methods and stability of bilirubin solutions. Clin. Chem., 19: 984 (1973).

5. Ebbesen. F.: Determination of serum bilirubin concentration during phototherapy of newborns and in vitro: results compared by the direct spectrometric method and the diazo method. Clin. Chem.. 23: 695 (1977).

6. Gambino, S. R. Bilirubin (modified Jendrassik and Grof)-Provisional. Standard Methods of Clinical Chemistry, Vol. 5, pp. 55-64. (Academic Press. New York, 1965).

7. Hanley, E. J., and DeWitt, D. P.. Physical Model for the Detection of Neonatal Jaundice by Multispectral Skin Reflectance Analysis, Proceedings of the Sixth Annual New England Bioengineering Conference, Kingston, R.I., pp. 346349, (Pergamon Press, New York, 1978).

8. Hannemann, R. E., DeWitt, D. P.. and Wiechel, J. F.: Neonatal serum bilinubin from skin reflectance. Pediatr. Res., 12: 207 (1978).

9. Hertz. H.. Dybkaer, R., and Lauritzen. J.: Direct spectrophotometric determination of the concentration of bilirubins in serum. Scand. J. Clin. Lab. Invest.. 33: 215 (1974).

10. Jacques, J. A., and Kuppenheim, H. F.: Spectral reflectance of human skin in the region 235-1000 mu. J. Appl. Physiol., 7: 523 (1955).

II. Krauss, A. N., Post, P. W.. Waldman, S., and Auld, P. A. M.: Skin reflectance in the newborn infant. Pediatr. Res., 10: 776 (1976).

12. Kubelka, P., and Munk, F.: Ein Beitrag zur Optik der Farbanstriche. Z. Techn. Physik, 12: 593 (1931)

13. Kuppenheim, H. F.. and Heer, R. R.: Spectral reflectance of white and negro skin between 400 and $1000 \mathrm{m \mu}$. J. Appl. Physiol., 4: 800 (1952).

14. Landowne, R. A.: Some experience with the precision performance of the Coulter chemistry system. Clin. Chem.. 23: 2176 (1977).

15. Lee. K., and Gartner, L. M.: Spectrophotometric characteristics of bilirubin Pediatr. Res., 10: 782 (1976).

16. Peevy, K. J., Mumford, L., Bruce, R., and Gross, S. J.: Estimation of serum bilirubin by spectral reflectance of the skin. Pediatr. Res., 12: 532 (1978).

17. Sheard. C., and Brown. G. E.: The spectrophotometric analysis of the color of the skin. Arch. Int. Med., 38: 816 (1926)

18. Simmons, W. R., Wilkinson, S. R.. Zurney, W. C. and Kast, J. L.. "EXOSYS," LARS Program Abstract 5000, Laboratory for Application of Remote Sensing. (Purdue University, W. Lafayette, IN, Sept. 1975).

19. Standards and Recommendations for Hospital Care of Newborn Infants, Ed. 6. pp. 93-97, (American Academy of Pediatrics. Evanston. IL. 1977).

20. Van Assendelf, O. W.: Spectrophotometry of Hemoglobin Derivatives, pp. 5557. (Royal Vangorcum Lid.. The Netherlands, 1970).

21. Dr. Hannemann is a visiting Professor of Chemical Engineering and pediatrician.

22. Dr. DeWitt is a Professor of Mechanical Engineering.

23. Dr. Hanley is a graduate research assistant in Mechanical Engineering.

24. Dr. Schreiner is an Associate Professor of Pediatrics.

25. Ms. Bonderman is a research associate.

26. This research was supported by a grant from the National Institutes of Arthritis. Metabolism and Digestive Diseases ROI AMI 8871.

27. Requests for reprints should be addressed to: Professor R. E. Hannemann, M. D.. School of Chemical Engineering, Purdue University, West Lafayette. Indiana 47907 USA.

28. Received for publication September 19. 1978.

29. Accepted for publication December 21, 1978. 